International Mathematical Forum, Vol. 8, 2013, no. 3, 125 - 132

\title{
Superharmonic Functions Outside Negligible Sets
}

\author{
M. G. Al-Akhrass \\ Mathematics Department \\ Faculty of Science, UOS \\ Sharjah, UAE
}

\begin{abstract}
A study of quasi superharmonic functions in Brelot spaces is introduced. A characterization of quasi superharmonic functions, in Brelot spaces, in terms of harmonic and finely harmonic measures is given. We prove that a Borel function is quasi superharmonic function if and only if it is a lower envelope of a class of superharmonic functions.
\end{abstract}

Mathematics Subject Classification: Primary 31B05, Secondary 31D05

\section{Introduction}

Let $\Omega$ be a Hausdorff connected, locally compact, non-compact space satisfying the second axiom of countability. Suppose that there is a system of harmonic functions on $\Omega$ satisfying the Brelot axioms. More precisely, the system of the harmonic functions satisfies the sheaf property, there is a base of open set, called regular, where Dirichlet problem is solvable and the limit of an increasing sequence of harmonic function is either harmonic or identically infinity. Moreover we assume this system satisfies the domination principle for superharmonic functions (Axiom D). We assume that there is a potential $>0$ on $\Omega$. We also assume further that constants are harmonic. A Euclidean connected open set $\Omega \subset \mathbb{R}^{n}$ for $n \geq 3$ with the class of all functions that satisfy Laplace equation on $\Omega$ is an example of Brelot space satisfying the above assumptions.

A function $u: \Omega \longrightarrow(-\infty, \infty]$ is defined to be superharmonic if it is not identically $\infty$ and if

(a) $u$ is lower semicontinuous on $\Omega$, and 
(b) for each $x \in \Omega$ and each regular open set $\omega$ containing $x$, $u$ satisfies the inequality

$$
u(x) \geq \int u d \mu_{x}^{\omega} .
$$

Where $\mu_{x}^{\omega}$ is the harmonic measure at $x$ with respect to $\omega$.

The set of all superharmonic (respectively nonnegative superharmonic) function on $\Omega$ will be denoted by $S H(\Omega)$ (respectively $S H^{+}(\Omega)$ ).

Now we can ask what class of functions we get if either (a) or (b) in the above definition is relaxed or strengthened.

In [2] B. J. Cole and T. J. Ransford proved that: If $u$ is a locally lower bounded function, with some measurability assumption, defined on a Euclidean Green open set $\Omega \subset \mathbb{R}^{n}, n \geq 2$. Then $u$ is quasi superharmonic (i.e. $u(x) \geq$ $\int u d \mu$ for each $x \in \Omega$ and every Jensen measure $\mu$ for $x$ on $\Omega$ ) if and only if $u$ is the infimum of all superharmonic functions $v$, on $\Omega$, such that $v \geq u$.

The authors in a later paper [3] proved implicitly that in the above result, it is enough to consider the harmonic measures instead of all Jensen measures.

In this note, the above result is extended to a general set up of axiomatic Brelot spaces. A characterization of quasi superharmonic function is given in terms of finely harmonic measures. We kept the above assumptions on the function $u$, that is: $u$ is never $-\infty$, and $u$ is locally lower bounded. But we replace the measurability condition, which is in terms of analytic sets, by assuming that $u$ is a Borel function because it is more natural. However our starting point is more elementary and we derive the very important Cartan convergence theorem as a consequence.

\section{Preliminaries}

Let $E$ be a subset of $\Omega$ and $u$ be any function $\geq 0$ on $E$. The reduced function of $u$ relative to $E, R_{u}^{E}$ is defined as the following:

$$
R_{u}^{E}(x)=\inf \left\{u(x): u \in S H^{+}(\Omega), u \geq \varphi \text { on } E\right\} .
$$

and its lower semicontinuous regularization, $\hat{R}_{u}^{E}$, is called the Balayaged function of $u$ relative to $E$.

For a domain $\omega \subset \Omega$ such that $E \subset \omega$, the restriction of $R_{u}^{E}$ to $\omega$ is defined as the following:

$$
\left(R_{u}^{E}\right)_{\omega}=\inf \left\{u \in S H^{+}(\omega): u \geq \varphi \text { on } E\right\} .
$$


$R_{u}^{E}$ is harmonic in $\Omega \backslash \bar{E}$, increasing in $E$ and $u$, and subadditive in $u$. Moreover, if $\omega$ is a regular open set then $\hat{R}_{u}^{\omega^{c}}=R_{u}^{\omega^{c}}$ every where and

$$
R_{u}^{\omega^{c}}(x)= \begin{cases}\int u d \mu_{x}^{\omega}, & x \in \omega \\ u(x), & x \notin \omega .\end{cases}
$$

A set $E$ is a polar set in $\Omega$ if there exists superharmonic function $u \geq 0$, such that $u=\infty$ in $E$. Also, $E$ is a polar if and only if there exists a superharmonic function $u>0$ in $\Omega$ such that $\hat{R}_{u}^{E}(x)=0$ for some point $x \in \Omega$. For a polar set $E$ and a point $x \in \Omega$, there exist a nonnegative superharmonic function $u$ such that $u=\infty$ on $E \backslash\{x\}$ and $u(x)<\infty$. The term "quasi-everywhere means except for a polar set. For more details see [1].

If $A$ is a Borel subset of $\Omega$ and $\mu$ a finite positive regular measure on $\Omega$, then there exists a unique regular measure $\mu^{A}$ (called balayaged measure) such that

$$
\int u d \mu^{A}=\int R_{u}^{A} d \mu, \forall u \in S H^{+}(\Omega) .
$$

An important special case is the case when $\mu=\delta_{x}$ for some $x \in \Omega$. In this case $\delta_{x}^{A}$ is defined by the relation:

$$
\int u d \delta_{x}^{A}=R_{u}^{A}(x), \forall u \in S H^{+}(\Omega)
$$

In the case when $A=\Omega-\omega$, where $\omega$ is an open set containing $x$, the measure $\delta_{x}^{\Omega-\omega}$ is just the well known harmonic measure, $\mu_{x}^{\omega}$. For details, see [4].

The fine topology on $\Omega$ is the coarsest topology on $\Omega$ which is finer than the given topology and makes every superharmonic function continuous.

In equation (1), when $A=\Omega-\omega$, where $\omega$ is finely open set containing $x$, the measure $\delta_{x}^{\Omega-\omega}$ is the fine harmonic measure of $\omega$ at $x$, supported by $\partial_{f} \omega$ ( the fine boundary of $\omega$ ). A finely lower semicontinuous, extended real valued function $f$ on a finely open set $U \subset \Omega$, is called finely superharmonic in $U$ if $f>-\infty$, finite on a dense subset of $U$ and $\forall$ finely open set $V \subset \subset U$ we have the inequality

$$
f(x) \geq \int f d \delta_{x}^{U \backslash V}, \forall x \in U .
$$

Finally, we recall that the lower semicontinuous regularization of a function $u$, denoted by $\hat{u}$, is the greatest lower semicontinuous function $\leq u$. Moreover, for every point $x \in \Omega$ :

$$
\hat{u}(x)=\liminf _{y \rightarrow x} u(y)
$$

For details, see [5]. 


\section{$2.1 \quad$ Jensen measures}

Definition 2.1. Let $U$ be an open subset of $\Omega$ and $x \in U$. A Jensen measure for $x$ (with respect to $U$ ) is a Borel probability Radon measure $\mu$, supported by a compact subset of $U$, such that every superharmonic function $u$ on $U$ satisfies

$$
u(x) \geq \int u d \mu .
$$

The set of all Jensen measures for $x$ (with respect to $U$ ) is denoted by $J_{x}(U)$.

It clear from the definition that if $x \in \Omega$ and $U_{1}, U_{2}$ are two open sets in $\Omega$ such that $x \in U_{1} \subset U_{2}$ then $J_{x}\left(U_{1}\right) \subset J_{x}\left(U_{2}\right)$ because $S H\left(U_{2}\right) \subset S H\left(U_{1}\right)$.

Remark 2.1. If $E$ is a polar set in $\Omega, x \in \Omega$ and $\mu \in J_{x}(\Omega)$ then $\mu(E \backslash\{x\})=$ 0 . To see this let $u$ be a nonnegative superharmonic function in $\Omega$ such that $u=\infty$ on $E \backslash\{x\}$ and $u(x)<\infty$. Then

$$
\infty \cdot \mu(E \backslash\{x\})=\int_{E \backslash\{x\}} u d \mu \leq \int u d \mu \leq u(x)<\infty .
$$

For $x \in \Omega$, let $H_{x}(\Omega)$ be the set of all harmonic measures at $x$ with respect to the relatively compact open subsets of $\Omega$, i.e.

$H_{x}(\Omega)=\left\{\mu_{x}^{V}: V\right.$ is a relatively compact open set such that $\left.x \in V \subset \bar{V} \subset \Omega\right\}$. It is clear that $H_{x}(\Omega) \subset J_{x}(\Omega)$. Let $F H_{x}(\Omega)$ be the set of all finely harmonic measures at $x$ with respect to the relatively compact finely open subsets of $\Omega$, i.e.

$F H_{x}(\Omega)=\left\{\delta_{x}^{\Omega \backslash V}: V\right.$ is a relatively compact finely open set such that $\left.x \in V \subset \bar{V} \subset \Omega\right\}$.

Clearly, $H_{x}(\Omega) \subset F H_{x}(\Omega)$ since every open set is also fine open set.

\section{Quasi superharmonic functions}

Definition 3.1. An extended real valued Borel function $u: \Omega \rightarrow(-\infty, \infty]$ is said to be quasi superharmonic on $\Omega$ if it satisfies the following two conditions:

(i) $u$ is locally bounded below.

(ii) for each $x \in \Omega$ and each $\mu \in J_{x}(\Omega)$, u satisfies the inequality

$$
u(x) \geq \int u d \mu
$$


It can be easily seen from the definition that superharmonic functions are quasi superharmonic functions, and the limit of an increasing sequence of quasi superharmonic function is quasi superharmonic.

Lemma 3.1. Let $u$ be an extended real valued Borel function which is locally lower bounded. Suppose that for each $x \in \Omega$, u satisfies the following inequality:

$$
u(x) \geq \int u d \mu \forall \mu \in H_{x}(\Omega) .
$$

Then $\hat{u}$ is a superharmonic function and $u=\hat{u}$ quasi everywhere.

Proof. $\hat{u}$, is superharmonic function because $u$ is nearly superharmonic, see [1]. To show that $u=\hat{u}$ quasi everywhere we must show that the set $\{x: u(x)>$ $\hat{u}(x)\}$ is a polar set. Let us first assume that $u$ is bounded above. Since the set $\{x: u(x)>\hat{u}(x)\}$ can be written as a countable union of sets of the form $A=\{x: u(x)-\hat{u}(x)>a\}$, it is enough to prove that $A$ is polar. Because the set $A$ is Borel we only have to show that every compact set $K \subseteq A$ is polar. Let $K$ be a compact subset of $A$ and let $\omega$ be a regular open set such that $K \subset \omega \subset \bar{\omega} \subset \Omega$. The function $u$ is locally lower bounded therefore, there exists a positive number $A$ such that $u+A \geq 0$ in $\bar{\omega}$. So, without loss of generality we may assume that $u \geq 0$ on $\omega$ (otherwise we consider $u+A$ ). $\mu_{x}^{\omega-K} \in H_{x}(\Omega)$ for $x \in \omega-K$, hence inequality (3) implies that

$$
u(x) \geq \int u d \mu_{x}^{\omega-K} \forall x \in \omega-K .
$$

Let $p$ be a positive continuous potential such that $p \leq a$ on $K$. Then $u>p+\hat{u}$ on $K$. Let

$$
f= \begin{cases}p+\hat{u} & \text { on } K \\ 0 & \text { on } \partial \omega .\end{cases}
$$

$u \geq f$ on $\partial(\omega-K)$ (the support of $\left.\mu_{x}^{\omega-K}\right)$, therefore

$$
u(x) \geq \int u d \mu_{x}^{\omega-K} \geq \int f d \mu_{x}^{\omega-K}, \forall x \in \omega-K .
$$

Because the function $x \longmapsto \int f d \mu_{x}^{\omega-K}$ is continuous (in fact harmonic) in $\omega-K$,

$$
\hat{u}(x) \geq \int f d \mu_{x}^{\omega-K}=\left(\hat{R}_{\hat{u}+p}^{K}\right)_{\omega}(x), \forall x \in \omega-K .
$$

Hence

$$
\left(\hat{R}_{\hat{u}}^{K}\right)_{\omega} \leq\left(\hat{R}_{\hat{u}+p}^{K}\right)_{\omega} \leq \hat{u} \text { in } \omega-K
$$


Axiom D implies that $\left(\hat{R}_{\hat{u}}^{K}\right)_{\omega}$ is the greatest harmonic minorant of $\hat{u}$ in $\omega-K$, so inequalities (4) imply that

$$
\left(\hat{R}_{\hat{u}}^{K}\right)_{\omega}=\left(\hat{R}_{\hat{u}+p}^{K}\right)_{\omega}=\left(\hat{R}_{\hat{u}}^{K}\right)_{\omega}+\left(\hat{R}_{p}^{K}\right)_{\omega} \text { in } \omega-K .
$$

Therefore $\left(\hat{R}_{p}^{K}\right)_{\omega}=0$ in $\omega-K$. So $K$ is a polar set in $\omega$ and thus a polar set in $\Omega$. For the general case, let $u_{n}=\min \{u, n\}$. Then for each $n, u_{n}$ is a bounded above quasi superharmonic function. By the above argument, $\min \{\hat{u}, n\}=\hat{u}_{n}=u_{n}$ outside a polar set, say $E_{n}$. Let $E=\bigcup_{n} E_{n}$. The set $E$ is polar and $\forall n$ we have $\hat{u}_{n}(x)=u_{n}(x) \forall x \notin E$, which implies that $\lim _{n} \hat{u}_{n}(x)=\lim _{n} u_{n}(x) \forall x \notin E$. Hence $\hat{u}(x)=u(x) \forall x \notin E$.

Theorem 3.1. Let $u$ be an extended real valued Borel function which is locally lower bounded. Then the following are equivalent:

1. $u$ is quasi superharmonic.

2. For each $x \in \Omega$, u satisfies the following inequality:

$$
u(x) \geq \int u d \mu \forall \mu \in F H_{x}(\Omega)
$$

3. $\hat{u}$ is a superharmonic function and $u=\hat{u}$ quasi everywhere.

4.

$$
u=\inf \{v \in S H(\Omega): v \geq u\} .
$$

Proof. (1) $\Rightarrow(2)$ : It is enough to show that for $x \in \Omega, F H_{x}(\Omega) \subset J_{x}(\Omega)$.

In fact, for a relatively compact finely open set $V$ and $x \in V$, the measure $\delta_{x}^{\Omega \backslash V}$ it is compactly supported because it is supported by $\partial_{f} V \subset \partial V$. Moreover, if $u$ is a superharmonic function on $\Omega$. Then $u$ is finely superharmonic function and, by definition of finely superharmonic functions, $u$ satisfies the inequality

$$
u(x) \geq \int u d \delta_{x}^{\Omega \backslash V}
$$

Furthermore, $\delta_{x}^{\Omega \backslash V}$ is a probability measure because $1,-1$ are finely superharmonic functions satisfy the above inequality. Hence for $x \in \Omega$ we have the relation $F H_{x}(\Omega) \subset J_{x}(\Omega)$.

$(2) \Rightarrow(3)$ The fact that $H_{x}(\Omega) \subset F H_{x}(\Omega)$ and lemma 3.1 imply the result. 
$(3) \Rightarrow(4)$ : Suppose that $\hat{u}$ is a superharmonic function and $u=\hat{u}$ quasi everywhere. It is clear that $u \leq \inf \{v \in S H(\Omega): v \geq u\}$. So we only need to show that $u(x) \geq \inf \{v(x): v \in S H(\Omega), v \geq u\} \forall x \in \Omega$. Let $x \in \Omega$. If $u(x)=\infty$ then there is nothing to prove, so assume that $u(x)<\infty$.

$\hat{u}$ is a superharmonic function and the set $E=\{y \in \Omega: \hat{u}(y)<u(y)\}$ is a polar set. Let $\epsilon>0$ and $w$ be a nonnegative superharmonic function such that $w=\infty$ on $E \backslash\{x\}$ and $w(x)<\epsilon$. There are two cases to be considered: $x \notin E$ or $x \in E$. If $x \notin E$, i.e. $\hat{u}(x)=u(x)$, then the function $\hat{u}+w$ is superharmonic $\hat{u}+w$ is $\geq u$, which implies that $\inf \{v \in S H(\Omega): v \geq u\} \leq \hat{u}+w$. In particular,

$$
\inf \{v(x): v \in S H(\Omega), v \geq u\} \leq \hat{u}(x)+w(x) \leq \hat{u}(x)+\epsilon .
$$

As $\epsilon$ is arbitrary, we get that

$$
\inf \{v(x): v \in S H(\Omega), v \geq u\} \leq \hat{u}(x)=u(x) .
$$

If $x \in E$, then $u(x)-\hat{u}(x)>0$. Let $p$ be a continuous positive potential such that at the point $x, p(x)=u(x)-\hat{u}(x)$. The function $\hat{u}+w+p$ is a superharmonic function $\geq u$. So,

$$
\inf \{v \in S H(\Omega): v \geq u\} \leq \hat{u}+w+p
$$

and in particular, at $x$

$$
\inf \{v(x): v \in S H(\Omega), v \geq u\} \leq \hat{u}(x)+w(x)+p(x) \leq u(x)+\epsilon .
$$

As $\epsilon$ is arbitrary we have

$$
\inf \{v(x): v \in S H(\Omega), v \geq u\} \leq u(x)
$$

$(4) \Rightarrow(1)$ Suppose $u=\inf \{v \in S H(\Omega): v \geq u\}$. Let $x \in \Omega$ and $\mu \in J_{x}(\Omega)$. Let $v \in S H(\Omega)$ such that $v \geq u$. Then $\int v d \mu \geq \int u d \mu$. The fact that $v$ is a superharmonic function implies $v(x) \geq \int v d \mu \geq \int u d \mu$. Hence

$$
v(x) \geq \int u d \mu \forall v \in S H(\Omega) \text { such that } v \geq u \text {. }
$$

Taking the infemum over all such $v^{\prime} s$ implies that $u(x) \geq \int u d \mu$. This proves (1). The proof is completed. 


\section{References}

[1] Brelot, M., Lectures on Potential Theory, Tata Institute, No 19, Bombay 1960, re-issude 1967.

[2] Cole, B.J. and Ransford, T. J., Subharmonicity without upper semicontinuity, J. Funct. Anal. 147(1997), 420-442.

[3] Cole, B.J. and Ransford, T. J., Jensen measures and harmonic measures, J. Reine Angew. Math. 541(2001), 29-53.

[4] Costantinescu, C. and Cornea, A., Potential theory on Harmonic Spaces, Grundlehern Math. Wiss. 158, Springer, New York, 1972.

[5] Fuglede, B., Finely Harmonic Function, Lecture Notes in Math. 289, Springer, Berlin-Heidelberg, 1972.

Received: September, 2012 